\title{
Improving Angular Resolution of the Crystal Orientation Determined with Spot Diffraction Patterns.
}

\author{
E.F. Rauch and M. Véron
}

\section{SIMaP, Grenoble INP - CNRS-UJF, BP 46 - 38402 Saint Martin d'Hères CEDEX, France}

The diffraction patterns collected on a Transmission Electron Microscope (TEM) contain the information related to the phase as well as the crystallographic orientation of the diffracting material. It is now possible to extract automatically both features from the pattern with a dedicated software that makes use of a template matching algorithm [1,2]. Basically, the idea is to invert the identification problem by comparing the collected image to templates that were pre-calculated for all possible orientations of the expected phases rather than deducing ab initio the crystallographic planes that may be related to the observed diffracting spots. This approach may be easily extended to any kind of crystallographic structure by adapting the template generation software. Template matching identification routines were recently coupled to electron precession and leads to a phase and orientation indexing tool that proves fast and efficient [3].

When compared to Kikuchi patterns, as the ones used in electron backscatter diffraction (EBSD) attachment for scanning electron microscopes (SEM), the Bragg spot patterns are considered as being less precise in terms of angular resolution. This comes from the fact that the positions of the Kikuchi lines are known to be very sensitive to the crystal orientation so that typically a $1^{\circ}$ resolution is easily achievable [3]. By contrast, for small change in orientation, the spot pattern looks self-similar i.e.: the diffracting beam position is fixed except of course for an azimuthal rotation of the crystal. Nevertheless, intensities are modified and the related sensitivity of the spot patterns is therefore questionable. The present work focused on the angular accuracy of the crystal orientation determination from Bragg spot diffraction patterns..

The intensity of a diffracting beam is strongly related to the Bragg condition through the excitation error. Patterns related to slightly misoriented crystals will exhibits small discrepancies. This is illustrated with the two theoretical patterns in figures 1.a and 1.c that were calculated for orientations close to the one corresponding to the diffraction image collected on an austenite grain (Fig. 1.b). The $0.5^{\circ}$ tilt between the templates is sufficient to promote visible decreases or increases in Bragg spot intensities, which are represented by the disk diameters, depending on the location of the reciprocal lattice nodes with respect to the Ewald sphere.

The sensitivity of the intensity changes may be used to refine the grain orientation estimate. To do so, the orientation resulting from the above mention template matching process is considered as a first guess. This orientation is then optimized by interpolating solutions that were deduced from the initial guess using a simplex algorithm.

The resulting orientation resolution is inferred by comparing successive measurements performed on deformed crystals. In figure 2.a, a twin produced by laser pulse deformation in pure iron intercept a grain boundary and produces local plastic deformation in the neighbor grain. The corresponding misorientations are detected by indexing all the spot diffraction patterns collected over an area that cover the twin and the intercepting grain boundary. The twinned grain normal orientation is close to [013] while the neighbor grain one is nearly [111]. The resolution of the output of the indexing software cannot be better than typically two times the orientation step used to generate the templates. Consequently a $1^{\circ}$ angular accuracy is hardly obtained in usual processing. Using the orientation refining algorithm, the resolution in figure 2 is 0.8 degree in the [111] and better than $0.3^{\circ}$ in the 
[013] grain (Fig. 2.b). The grain orientation dependency of the angular precision is related to the fact that the second orientation exhibits a $180^{\circ}$ ambiguity that makes it harder to identify [4]. It is worth mentioning that these results were obtained with diffraction images collected with an external camera focused on the phosphorus screen through the TEM window and, moreover, with a signal binned down to $150 \times 150$ pixels in order to promote a faster acquisition rate $(20 \mathrm{fps})$. Consequently a better angular resolution is probably achievable with a less crude acquisition procedure. The present results suggest that Bragg spot intensities may be as sensitive as Kikuchi lines positions for orientation determination.

\section{References}

[1] E.F. Rauch, and L. Dupuy, L.: Arch. Metall. Mater 50 (2005) 87-99

[2] E.F. Rauch and M. Véron, J. Mater. Sci. Eng. Tech. 36 (2005) 552-556.

[3] F.J. Humphreys, Scripta Mater 51 (2004) 771-776

[4] E.F. Rauch at al., Special Issue on "Precession Electron Crystallography" of Zeits. Krist. 225, issue 2-3, 2010, in press

[5] A. Morawiec, and E. Bouzy, J. Appl. Cryst. 39 (2006) 101-103.
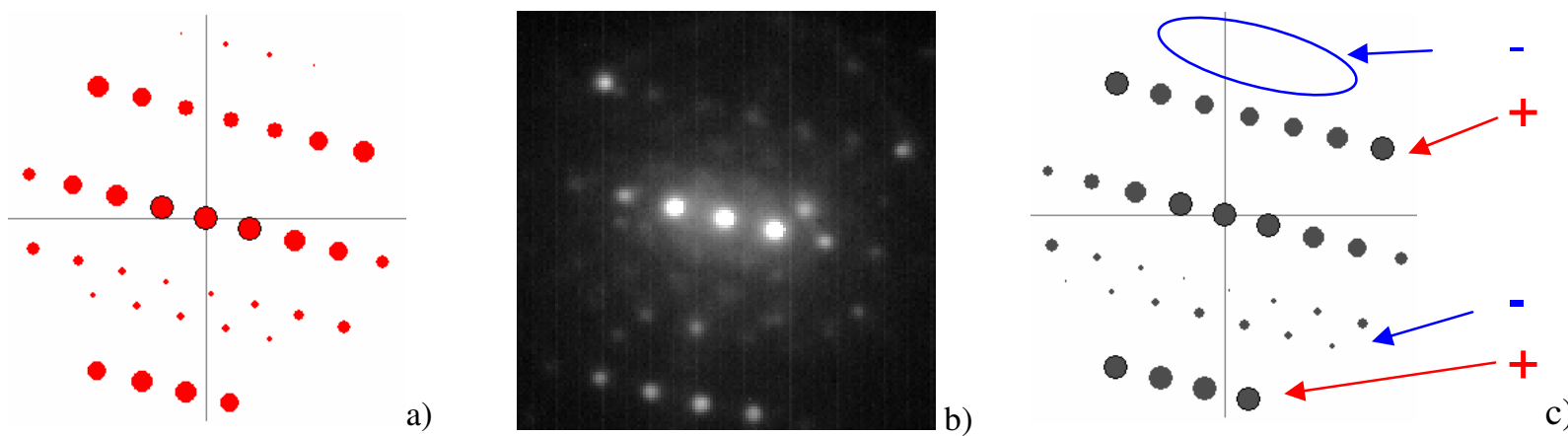

FIG. 1. Calculated diffraction patterns $(\mathrm{a}, \mathrm{c})$ for orientations close to the one corresponding to the diffracting austenite crystal in (b). The spot intensities in (c) increases (+) or decreases (-) for a $0.5^{\circ}$ tilt with respect to (a) depending if the lattice planes approach or depart from the Bragg conditions.
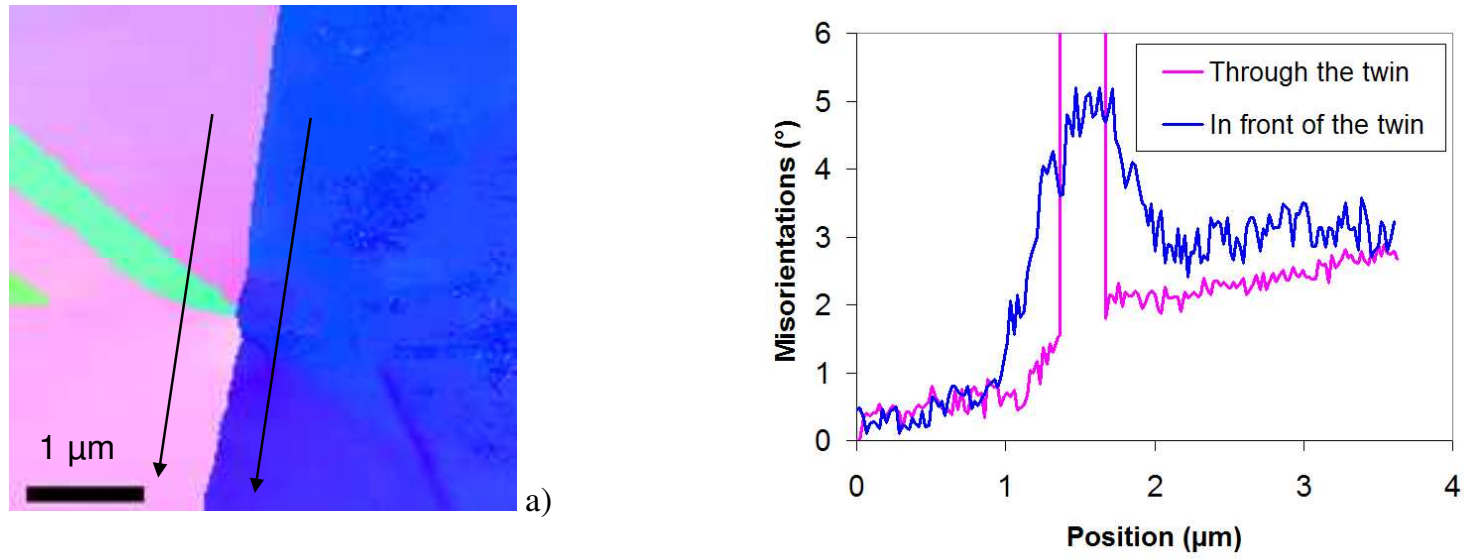

b)

FIG. 2. a) a twin crossing the left grain is interacting with the right grain producing a local misorientation. b) the angular resolution achievable with spot diffraction patterns is lower than $1^{\circ}$ 